\title{
COMPETITION DEPENDING ON LITTER SIZE IN THE MOUSE
}

\author{
PETER HULL \\ Department of Genetics, University of Liverpool
}

Received I 4.vii.66

\section{INTRODUCTION}

IN a stock of mice where the genes $c$ and $c^{c h}$ were segregating the ratio of homozygous to heterozygous animals in early litters seemed to differ from that in later litters. Therefore, a study was made of the relationship between ratio of genotypes in a litter and the parity or age of the mother or the size of the litter, which is known to have a marked relation with maternal age (e.g. Murray, 1934).

\section{EXPERIMENTAL MATERIAL}

The mice were derived from a cross between two inbred strains LAN and I 29/J. The first was homozygous $c c$ while in the second strain the allelic genes $c$ and $c^{c h}$ were segregating. The gene $p$ (pink eye), homozygous in $129 / \mathrm{J}$ was eliminated from the stock by mating the original crossbred animals among themselves and testing female progeny for homozygosity of wild type at the $p$ locus. These females were then back-crossed to LAN males. Progeny from these matings were intercrossed. Animals for the experimental crosses were obtained either from the progeny of these matings or after one further generation. Because of the way the foundation crosses were made there was a probability of more than 95 per cent. that a $c$ gene in the experimental stock was derived from the LAN strain. The $c^{c h}$ genes must of course have come from $129 / \mathrm{J}$.

Single pair matings were set up and the mice remained together throughout the period of the experiment. Litter size was scored at birth: it was also possible to distinguish albino young from their litter mates with pigmented eyes at this time. The full classification of the progeny was made at three weeks of age, when the young were weaned. The average age of females at mating was $67 \cdot 1$ days. Twentythree fertile matings of $c c$ female with $c c^{c h}$ male, I9 of $c c^{c h}$ female with $c c$ male, 23 of $c c^{c h}$ female with $c^{c h} c^{c h}$ male and 18 of $c^{c h} c^{c h}$ female with $c c^{c h}$ male were obtained. These were continued until either six litters had been produced or the mating had lasted 230 days. At this point the mating was terminated.

\section{RESULTS}

Figure I gives the mean ratio of heterozygotes to total littermates for first to sixth litters, together with the number of litters of each type. There is some indication that there is a small increase in the proportion of heterozygotes with increasing parity (in fact the regressions of ratio on litter number which can be calculated are all positive-see table I). Figure 2 shows the data rearranged with the mean ratio of heterozygotes given for the various litter sizes, firstly for the mating of $c c$ females with $c c^{c h}$ males plus $c c^{c h}$ females with $c c$ males and, secondly, for the two reciprocal mating types of $c c^{c h}$ and $c^{c h} c^{c h}$. 


\section{INTERPRETATION OF DATA}

Since in each type of mating the two classes of offspring, heterozygous $\left(c c^{c h}\right)$ or homozygous (either $c c$ or $c^{c h} c^{c h}$ ) could be produced, the dependent variable considered was the ratio:

$$
y=\frac{\text { heterozygous littermates }}{\text { heterozygous plus homozygous littermates }} \text {. }
$$
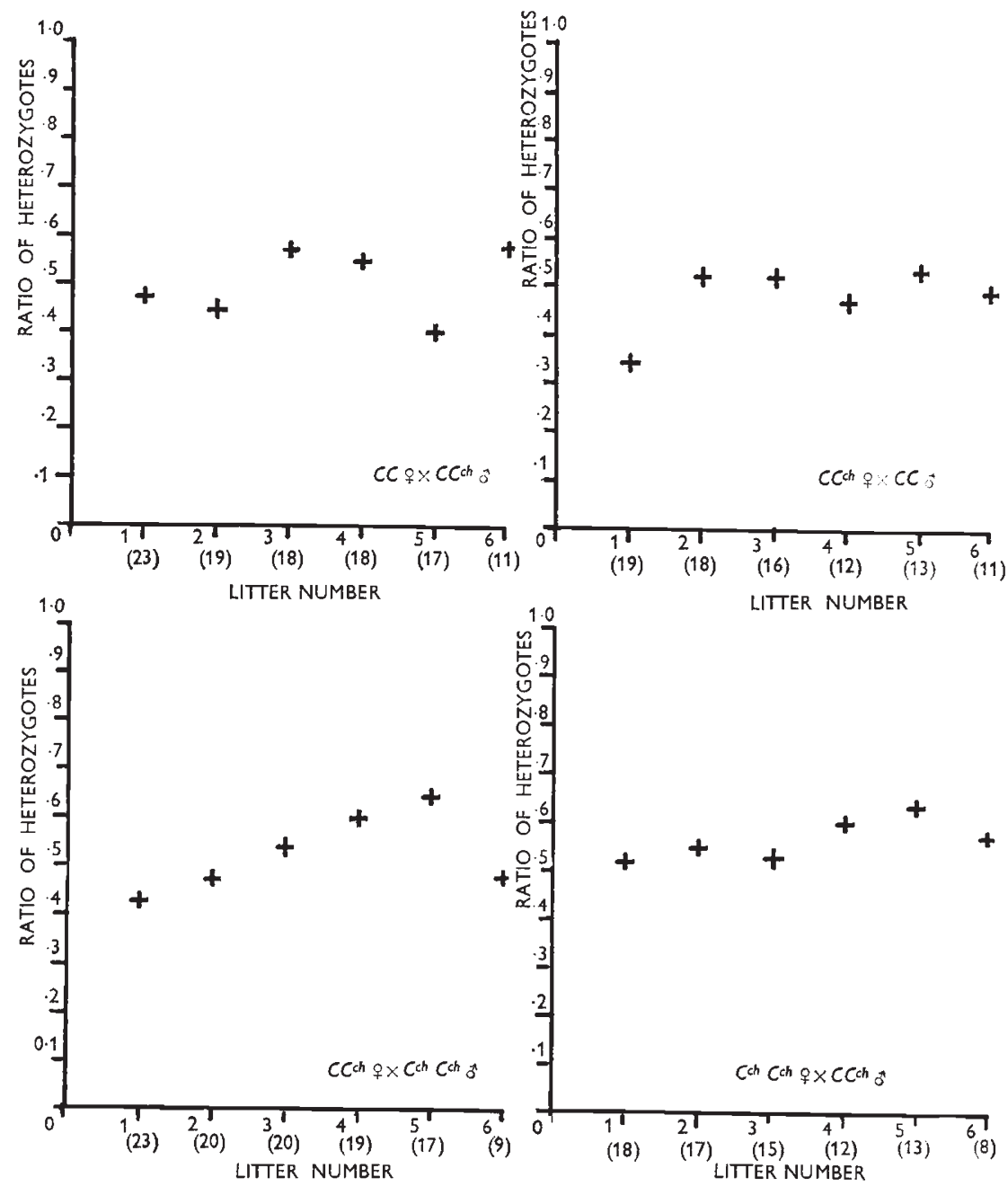

FIG. 1.-Relationship between ratio of heterozygotes and litter number (parity) for the four types of cross. Number of litters in brackets.

It was found that this variable had a distribution not significantly different from normality. No transformation was considered necessary: this variable gave the most conservative estimate of the significance of the coefficient calculated. The three independent variables were then: litter number (parity) i.e. first, second, etc., litter from a particular 


\begin{tabular}{|c|c|c|c|c|c|}
\hline \multirow{9}{*}{ 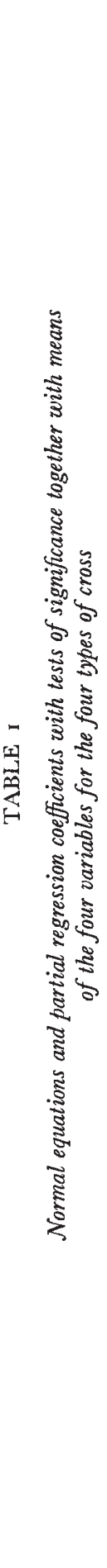 } & & 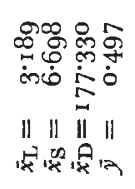 & 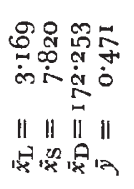 & 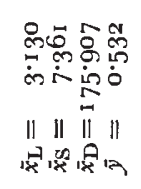 & 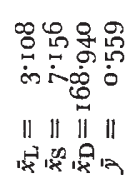 \\
\hline & $A$ & 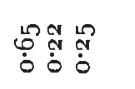 & 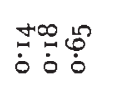 & 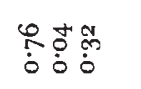 & 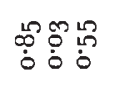 \\
\hline & + & 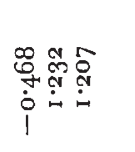 & 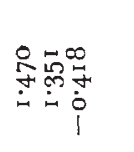 & 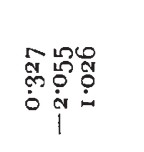 & 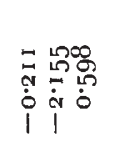 \\
\hline & 0 & 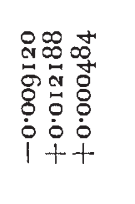 & 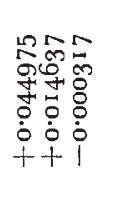 & 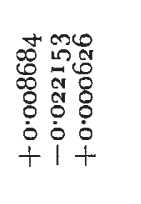 & 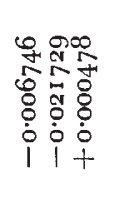 \\
\hline & $\lambda$ & 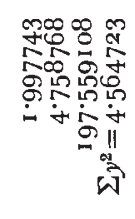 & 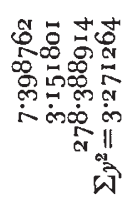 & 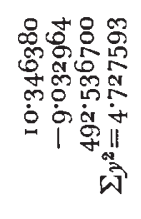 & 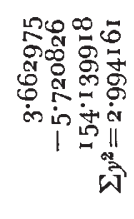 \\
\hline & & 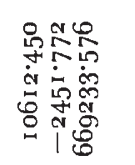 & 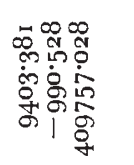 & 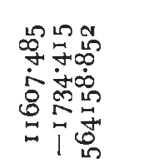 & 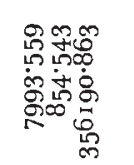 \\
\hline & 易 & 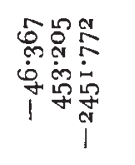 & 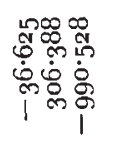 & 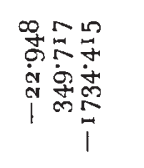 & 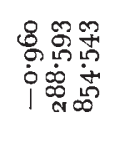 \\
\hline & & 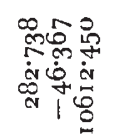 & 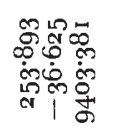 & 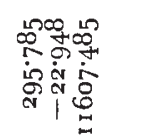 & 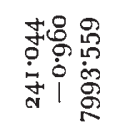 \\
\hline & 总 & 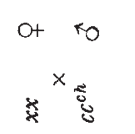 & $\begin{array}{l}\text { Ot ro } \\
\underbrace{\circ} 8\end{array}$ & 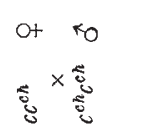 & 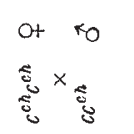 \\
\hline
\end{tabular}


mating, $\left(x_{\mathrm{L}}\right)$; litter size, i.e. number of living young born $\left(x_{\mathrm{s}}\right)$ and age, in days, of the mother at the time of the birth of the litter, $\left(x_{\mathrm{D}}\right)$. Partial regressions of ratio on each one of the variables, independent of the
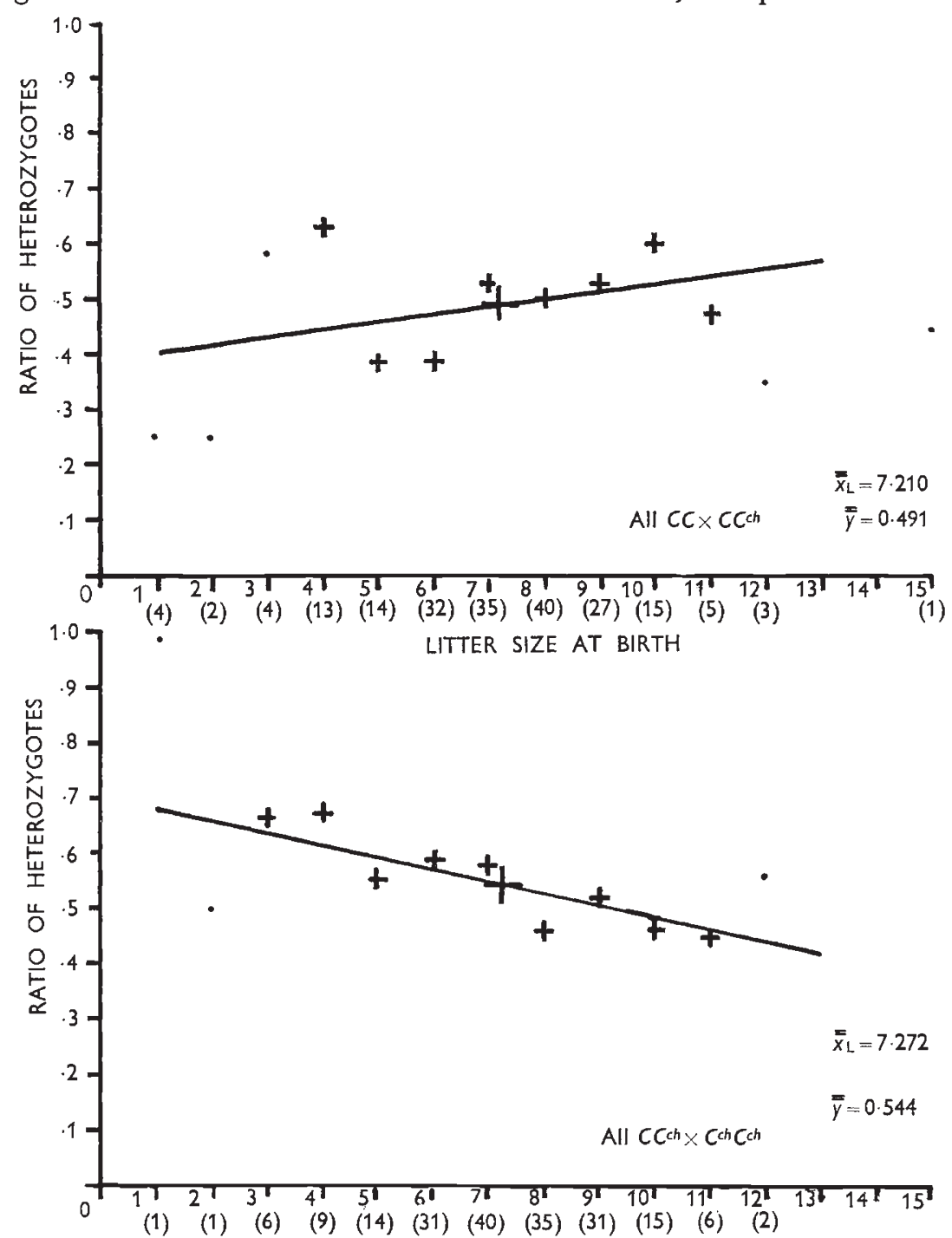

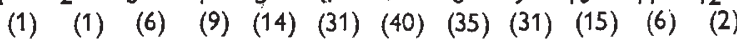

LITTER SIZE AT BIRTH

FIG. 2.-Relationship between ratio of heterozygotes and litter size for pooled reciprocal matings of $c c$ with $c c^{c h}$ or of $c c^{c h}$ with $c^{c h} c^{c h}$. Means based on five or more litters, crosses; means based on less than five litters, single points. Number of litters in brackets.

other two, were calculated from the corrected sums of squares and cross products using an electronic computer. Table I gives first the values of

$$
\mathrm{LSD}=\left(\begin{array}{lll}
\Sigma x_{\mathrm{L}}^{2} & \Sigma x_{\mathrm{L}} x_{\mathrm{s}} & \Sigma x_{\mathrm{L}} x_{\mathrm{D}} \\
\Sigma x_{\mathrm{S}} x_{\mathrm{L}} & \Sigma x_{\mathrm{S}}^{2} & \Sigma x_{\mathrm{S}} x_{\mathrm{D}} \\
\Sigma x_{\mathrm{D}} x_{\mathrm{L}} & \Sigma x_{\mathrm{D}} x_{\mathrm{S}} & \Sigma x_{\mathrm{D}}^{2}
\end{array}\right)
$$


and

$$
\begin{aligned}
& y=\left(\begin{array}{c}
\Sigma x_{\mathrm{L}} y \\
\Sigma x_{\mathrm{s} y} \\
\Sigma x_{\mathrm{D} y}
\end{array}\right), \\
& b=\left(\begin{array}{l}
b_{\mathrm{YL} \cdot \mathrm{sD}} \\
b{ }_{\mathrm{Ys} \mathrm{L} \mathrm{D}} \\
b{ }_{\mathrm{YD} \cdot \mathrm{L} \mathrm{s}}
\end{array}\right),
\end{aligned}
$$

obtained from: $\operatorname{LSD}^{-1} y=b$, together with their standard errors and the probability, given by a $t$-test, of obtaining the observed values by chance for each type of cross. Also included in table I are $\Sigma y^{2}$ and the mean values: $\bar{x}_{\mathrm{L}}, \bar{x}_{\mathrm{S}}, \bar{x}_{\mathrm{D}}$ and $\bar{y}$. There is a significant negative partial regression of ratio on litter size in the reciprocal matings of $c c^{c h} \times c^{c h} c^{c h}$ : in small litters there is an excess of heterozygotes. There is no similar relationship in reciprocal matings of $c c$ and $c c^{c h}$. Lines of slope $b=+0.013$ or $b=-0.022$ are drawn in fig. 2 .

\section{DISCUSSION}

Since there is a significant increase in the proportion of $c c^{c h}$ heterozygotes to $c^{c h} c^{c h}$ homozygotes in smaller litters, regardless of whether the mother is $c c^{c h}$ or $c^{c h} c^{c h}$ there is evidence of an interaction between offspring of different genotypes, rather than between specific maternal and fotal genotypes. Size of litter could depend on two factors: (a) number of viable zygotes present at implantation, a reflection to some extent of the number of eggs produced at an ovulation, or $(b)$ the number of offspring that the female is capable of carrying through to birth at that time.

There is evidence (Macdowell and Lord, I925), that ovarian activity increases in female mice of several strains at least up to the tenth litter, measured by the average number of corpora lutea counted in living mice at successive pregnancies. The increase may be more rapid over the first three pregnancies, but appears to continue fairly uniformly thereafter at a slower rate. At any rate there is no decline in production of corpora lutea from the fourth or fifth litter, when the total number of living young at parturition would be declining. In attempting to account for the observed relationship between genotypic ratio and litter size we are thus left with two possible explanations. It might be that the heterozygote is at an advantage when only a few eggs are shed, but that in conditions where the embryos are more crowded and competition for available resources becomes more severe the $c^{c h} c^{c h}$ homozygotes are at an advantage. On the other hand, if small litters are predominantly a result of physiological inadequacy of the mother at this time, this would suggest that $c c^{c h}$ offspring are at an advantage under these circumstances and that this advantage declines when available resources are greater. If we calculate the weighted total regression of litter size on litter number during the period of the experiment we have: $b_{\mathrm{s} \cdot \mathrm{L}}=-0 \bullet 066$. Average litter size in plotted against 
litter number in fig. 3 , where there is little indication of an increase in litter size at the beginning of the reproductive life of the females.

One important difference between the conditions of this test and those of Murray's investigation is that while his mice were mated at the age of one month, the average age of females at mating in this test was just over 2 months. It may be that this delay was such that the females had reached somewhere near their physiological optimum by the time

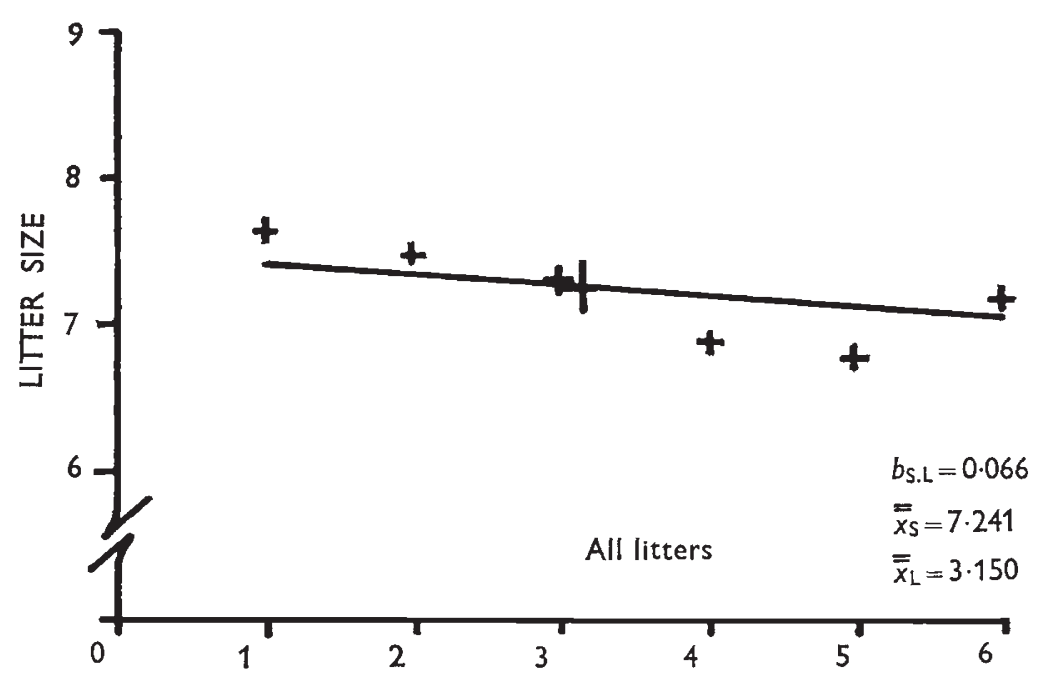

LITTER NUMBER

FIG. 3--Relationship between litter size and litter number: pooled data from all four crosses.

they were mated and that differences in litter size reflect the slow decline associated with increasing age. In this case we would not have any comparison of the performance of the genotypes under conditions of low female fecundity and we are left with an indication of the superiority of the $c c^{c h}$ individuals when the physiological condition of the mother becomes less favourable.

The effect observed may not be due to the $c$ and $c^{c h}$ genes themselves but to closely linked genes, since the two genes came from different stocks and sufficient time had not elapsed for the genes of the test stock to have reached linkage equilibrium.

\section{SUMMARY}

I. An apparent increase in the numbers of heterozygous $c c^{c h}$ offspring compared with the numbers of their homozygous $c c$ or $c^{c h} c^{c h}$ littermates was noticed in late litters compared with early litters from the same matings.

2. There was a significant negative relationship between litter size and ratio $c c^{c h} /\left(c c^{c h}+c^{c h} c^{c h}\right)$ littermates. This was not found for the ratio $c c^{c h} /\left(c c^{c h}+c c\right)$ littermates. 
3. None of the partial regressions of ratio on age of female or of ratio on litter number was significantly different from zero.

Acknowledgments.-I wish to thank Dr D. S. Falconer and Professor P. M. Sheppard for their helpful criticism. I am grateful to the Computing Laboratory of the University of Liverpool for the opportunity to use the K.D.F.9 computer. Part of this work was done during the tenure of a D.S.I.R./N.A.T.O. Fellowship.

\section{REFERENCES}

MACDOWELL, E. C., AND LORD, E. M. 1925. The number of corpora lutea in successive pregnancies. Anat. Rec., 3I, I43-148.

MURRAY, w. s. r935. The breeding behaviour of the dilute brown stock of mice

(Little $d b a)$. Amer. F. Cancer, 2o, 373-593. 